\title{
Always consider left ventricular outflow tract obstruction in hemodynamically unstable patients
}

\author{
Antoine G. Rochon, MD • Philippe L. L'Allier, MD • \\ André Y. Denault, MD
}

Received: 11 June 2009/Accepted: 10 August 2009/Published online: 22 October 2009

(C) Canadian Anesthesiologists' Society 2009

Transesophageal echocardiography (TEE) plays a key role in the evaluation of hemodynamic instability, particularly in the diagnosis of left ventricular outflow tract obstruction (LVOTO) where it can completely alter patient management. The purpose of these rounds is to highlight how TEE can be instrumental in ensuring a timely diagnosis of this lesion by using examples from the recent archives of the Montreal Heart Institute of hemodynamic instability secondary to previously undiagnosed LVOTO. In some cases, TEE can also serve as an invaluable tool to evaluate the response to therapy in the perioperative and critical care settings. The Research Ethics Committee of the Montreal Heart Institute granted approval for use of the related images for research and publication purposes.

\section{Case 1}

An elderly male with coronary artery disease was scheduled for coronary artery bypass surgery. His primary

Electronic supplementary material The online version of this article (doi:10.1007/s12630-009-9174-y) contains supplementary material, which is available to authorized users.

A. G. Rochon, MD · A. Y. Denault, MD ( $\square)$

Department of Anesthesiology, Montreal Heart Institute, Université de Montréal, 5000 Belanger Street, Montreal, QC H1T 1C8, Canada

e-mail: andre.denault@umontreal.ca; denault@videotron.ca

P. L. L'Allier, MD

Department of Cardiology, Montreal Heart Institute, Université de Montréal, Montreal, QC, Canada

A. Y. Denault, MD

Division of Critical Care, Department of Medicine, Centre

Hospitalier de l'Université de Montréal, Montreal, QC, Canada symptom was dyspnea, and his medical history was notable for systemic hypertension. Following induction of general anesthesia, premature ventricular complexes were associated with a non-compensatory increase in arterial pressure (Fig. 1). His TEE exam revealed severe LVOTO with systolic anterior motion (SAM) of the anterior leaflet of his mitral valve (Figs. 1 and 7, Video \#1 and 2). Intravenous fluids and a direct alpha agonist were given.

\section{Case 2}

An elderly female without coronary artery disease was scheduled for aortic valve replacement. Her medical history included hypertension. Following aortic valve replacement, she became hemodynamically unstable. While her left ventricular function was normal, her TEE exam showed severe LVOTO (Fig. 2). The patient did not respond to medical therapy; she returned on cardiopulmonary bypass and underwent mitral valve replacement.

\section{Case 3}

A young male patient with Crohn's disease was admitted to the intensive care unit for management of septic shock. His heart rate was 120 beats $\cdot \min ^{-1}$ and his cardiac index was $3.4 \mathrm{~L} \cdot \min ^{-1} \cdot \mathrm{m}^{-2}$ with elevated filling pressures. He required intensive pharmacologic intervention to maintain his system blood pressure. In response to the findings on TEE examination (Fig. 3), $1000 \mathrm{~mL}$ of Ringer's lactate was infused and intravenous metoprolol was administered. As his heart rate (HR) gradually slowed, left ventricular filling pressures decreased gradually, as did the requirement for vasoactive support. His TEE exam showed evidence of LVOTO (Fig. 3, Video \#3). 


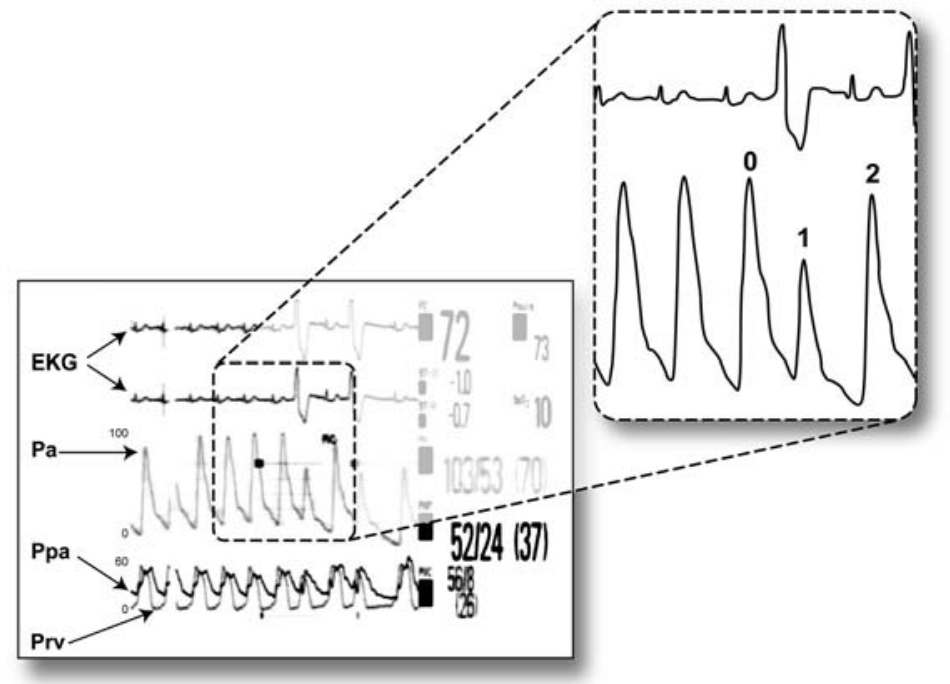

Fig. 1 Brockenbrough-Braunwald phenomenon. Hemodynamic waveforms in a hemodynamically unstable patient after the induction of anesthesia. Note that the arterial pressure $(\mathrm{Pa})$ of the beat $(0)$ before the premature ventricular complex (PVC) was followed by a second beat (1) with a normal lower systolic Pa. However, the following beat (2) was abnormally lower than the one prior to the PVC. This phenomenon is suggestive of a Brockenbrough-Braunwald phenomenon, which is pathognomonic of left ventricular outflow tract obstruction (LVOTO). This patient was found to have LVOTO on the echocardiographic exam (see Video \#1 and 2) (EKG electrocardiogram; Ppa pulmonary artery pressure; Prv right ventricular pressure)
A

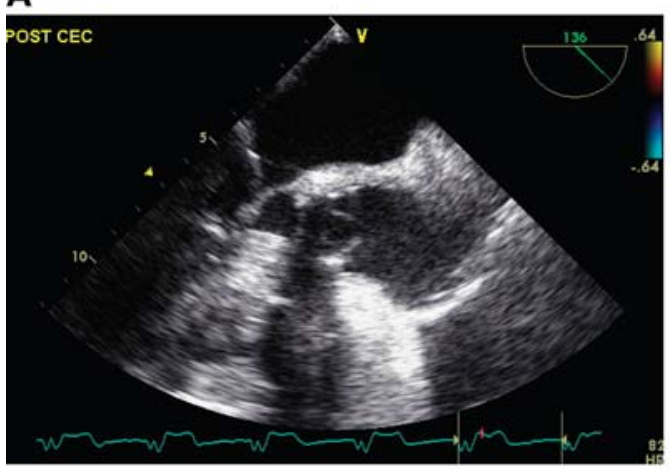

C

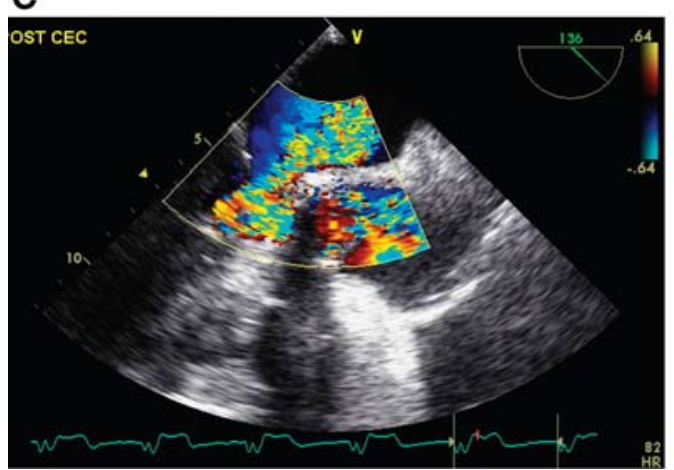

Fig. 2 Left ventricular outflow tract obstruction (LVOTO) with systolic anterior motion (SAM). a-c Mid-esophageal long-axis view in a patient with LVOTO after aortic valve replacement. During systole, the anterior leaflet of the mitral valve is dragged or pushed into the left ventricular outflow tract. This makes the mitral valve incompetent and leads to mitral regurgitation (MR). The term SAM describes the
B
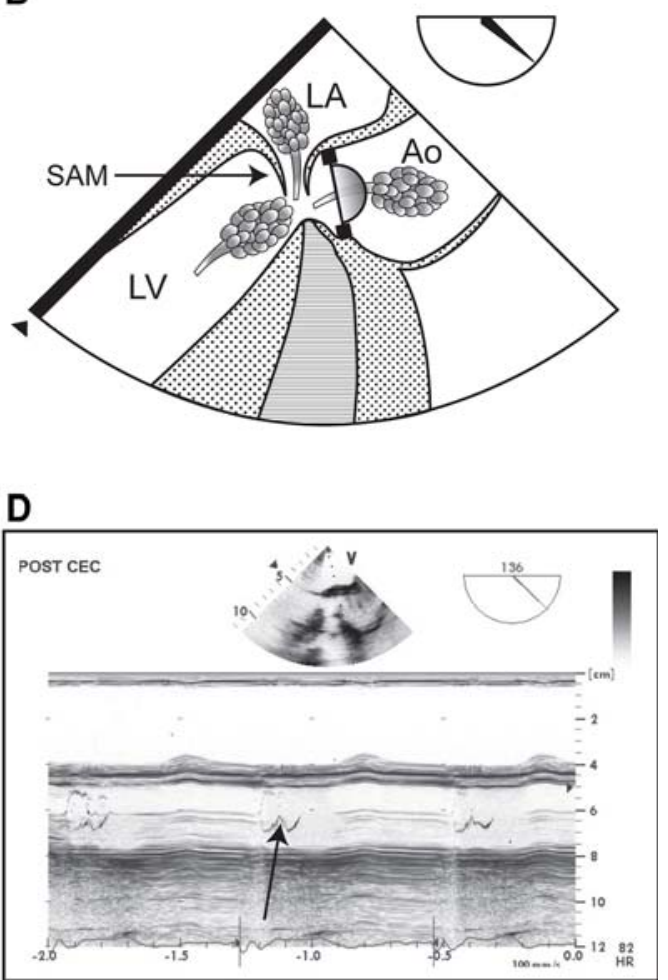

displacement of the anterior leaflet of the mitral valve in the left ventricular outflow tract. This results in LVOTO and incompetent mitral valvular function. d Using M-mode on the aortic valve, a midsystolic notch (arrow) is seen (Ao aorta; $L A$ left atrium; $L V$ left ventricle) 
Fig. 3 Dynamic left ventricular outflow tract obstruction. a, b Mid-esophageal long-axis view in a young man with septic shock and hemodynamic instability. Part of the anterior mitral valve leaflet is obstructing the left ventricular outflow tract. c, d This was associated with mitral regurgitation (MR). His hemodynamic condition improved with volume loading and $\beta$-adrenergic blockade (Ao aorta; $A o V$ aortic valve; $L A$ left atrium; $L V$ left ventricle; $S A M$ systolic anterior motion) (see Video \#3) (With permission of Denault et $\left.a l .{ }^{5}\right)$

Fig. 4 Left ventricular outflow tract obstruction (LVOTO) in a patient with right ventricular failure. a, b Mid-esophageal long-axis view in a patient with LVOTO in the intensive care unit after a cardiac arrest from an occluded right coronary artery. Severe LVOTO was associated with right ventricular failure. c Using continuous wave Doppler in the left ventricular outflow tract, a late systolic peak (dagger-shape) velocity can be obtained. From this velocity, the pressure gradient across the left ventricular outflow tract can be estimated (Ao aorta; $A o V$ aortic valve; $L A$ left atrium; $L V$ left ventricle; $M R$ mitral regurgitation; SAM systolic anterior motion)
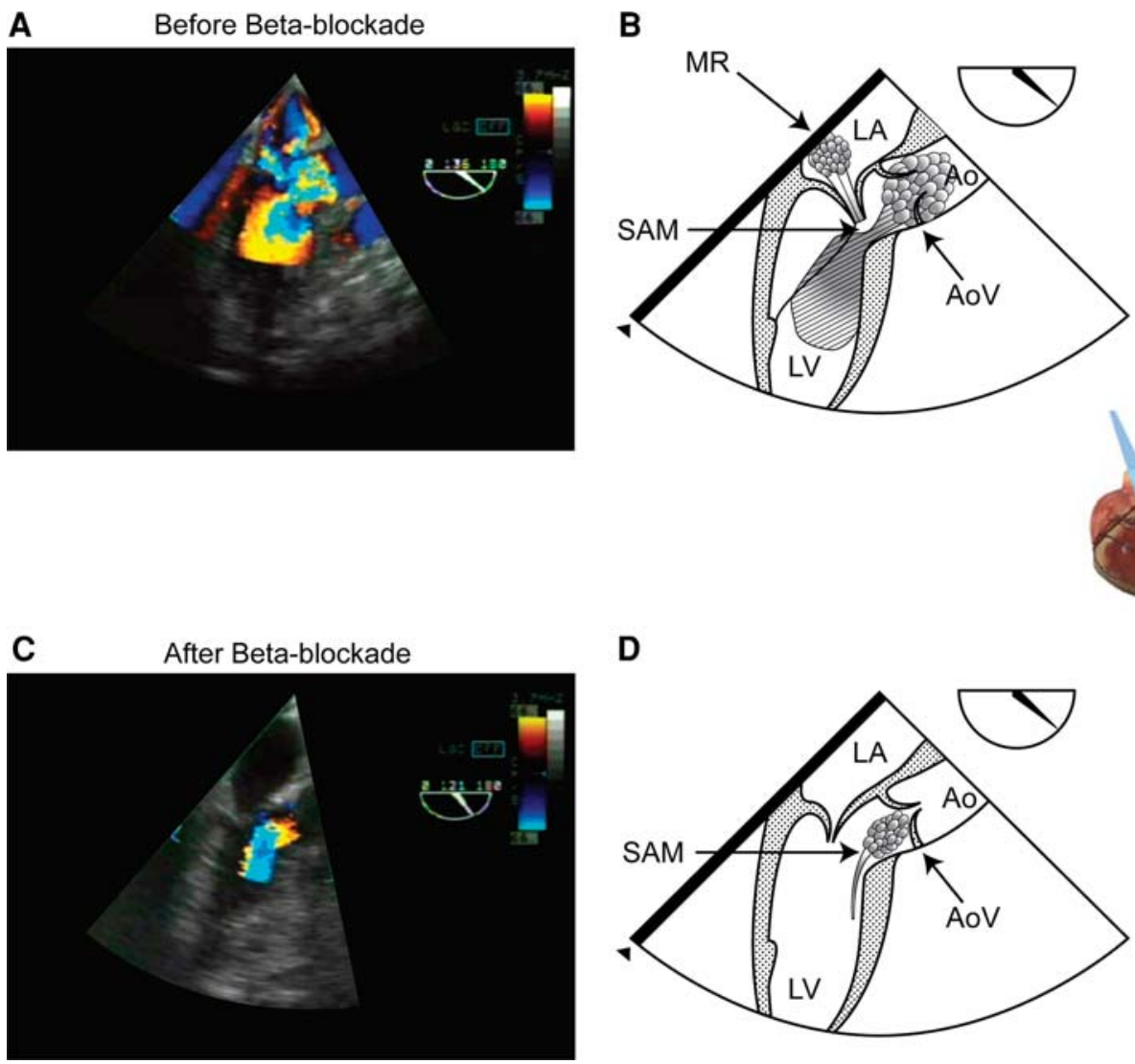

D
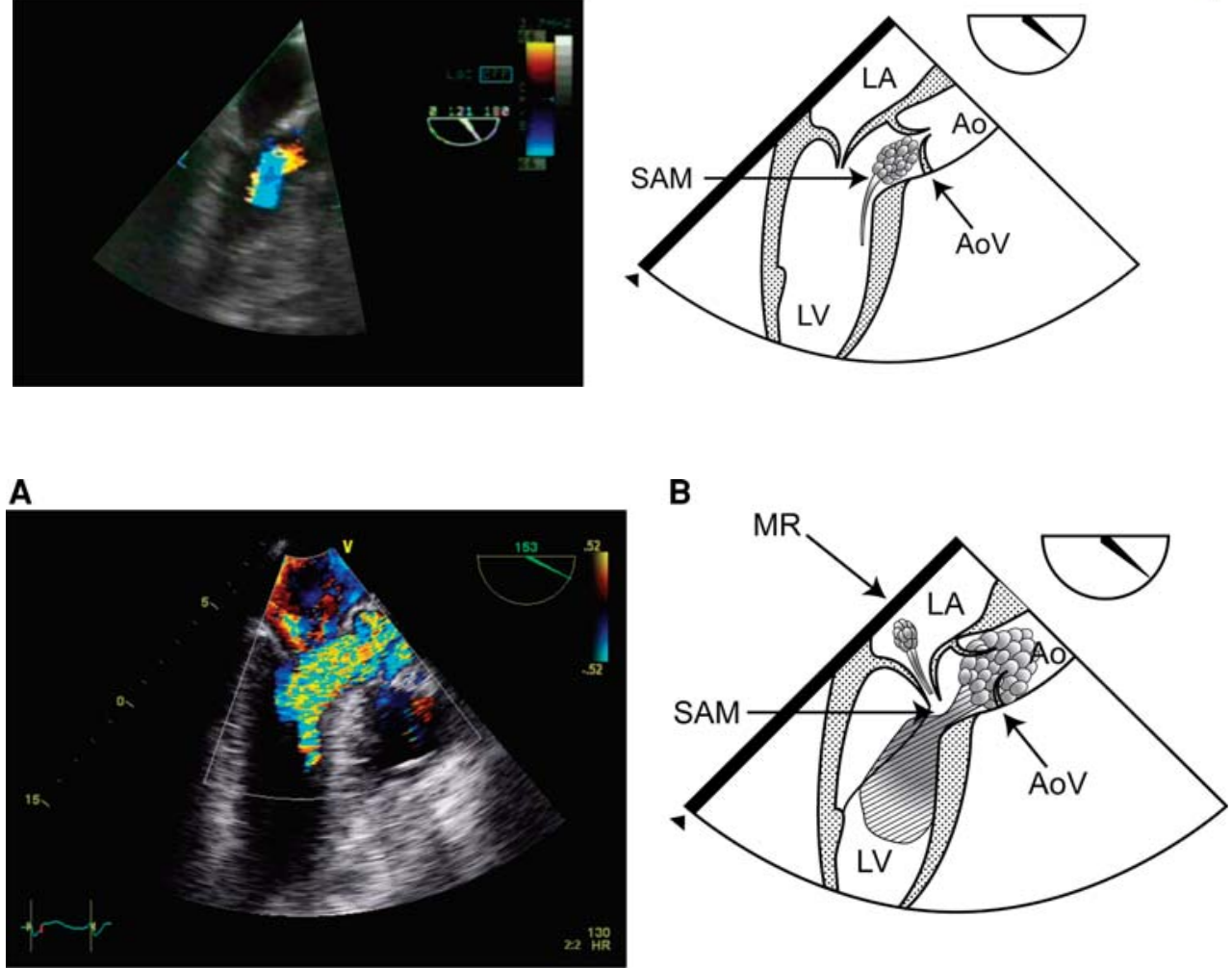
Fig. 5 Risk factors of systolic anterior motion (SAM): SPASM ( $L A$ left atrium; Ao aorta; $L V$ left ventricle; $R V$ right ventricle; $P M L$ posterior mitral leaflet length; $A M L$ anterior mitral leaflet length; $L V O T$ left ventricular outflow tract; $S L C L$ septal to leaflet coaptation length) (Adapted with permission of Denault et al..$^{5}$ )
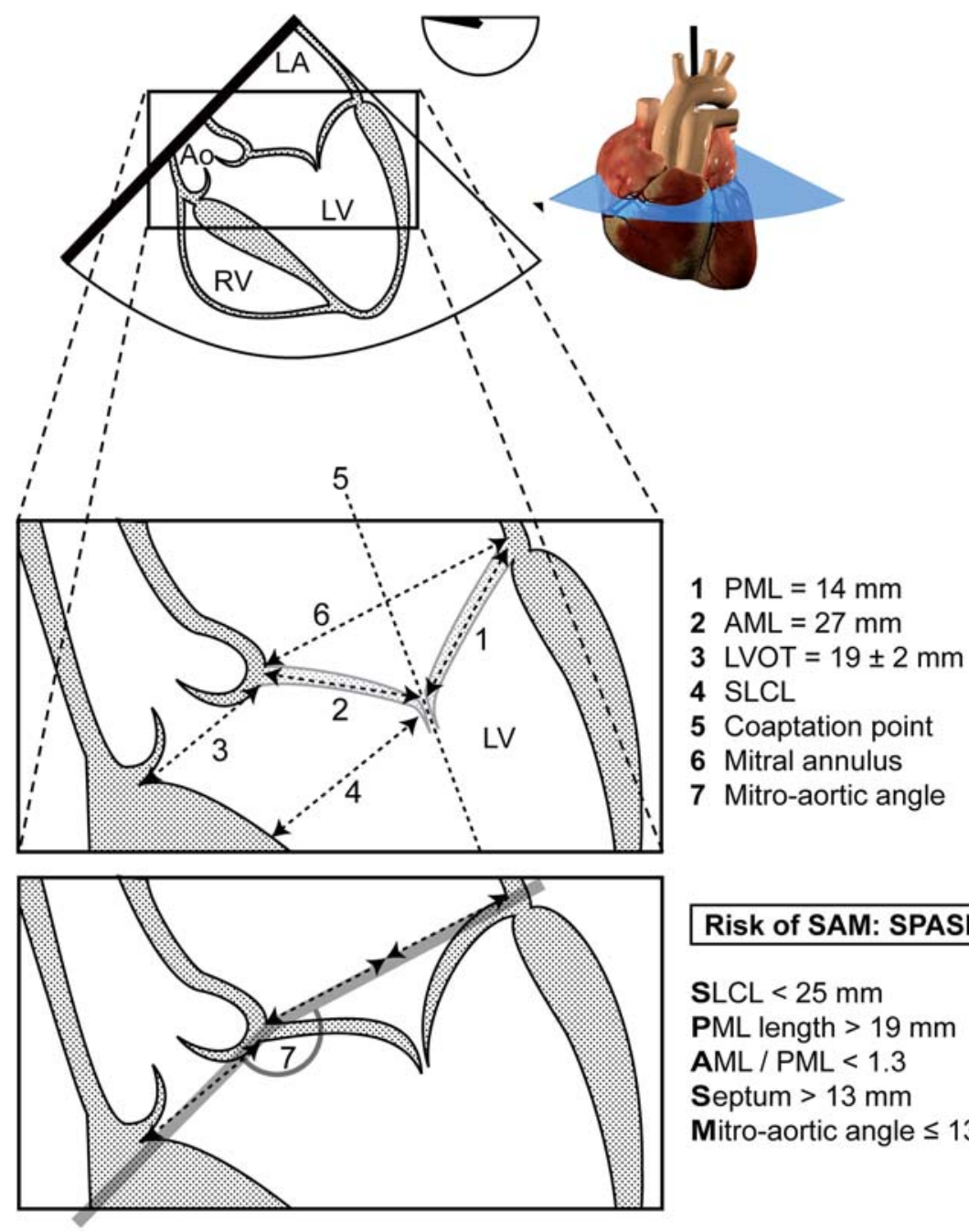

Risk of SAM: SPASM

$\mathrm{SLCL}<25 \mathrm{~mm}$

PML length $>19 \mathrm{~mm}$

AML / PML $<1.3$

Septum $>13 \mathrm{~mm}$

Mitro-aortic angle $\leq 130^{\circ}$
Fig. 6 Venous return (VR) and pressure-volume relationship (PVR) in left ventricular outflow tract obstruction (LVOTO). a In LVOTO, the VR will be reduced with associated elevated right atrial pressure (dotted lines). b On the PVR, the stroke volume will be reduced as a consequence of increased left ventricular pressure and mitral regurgitation
A

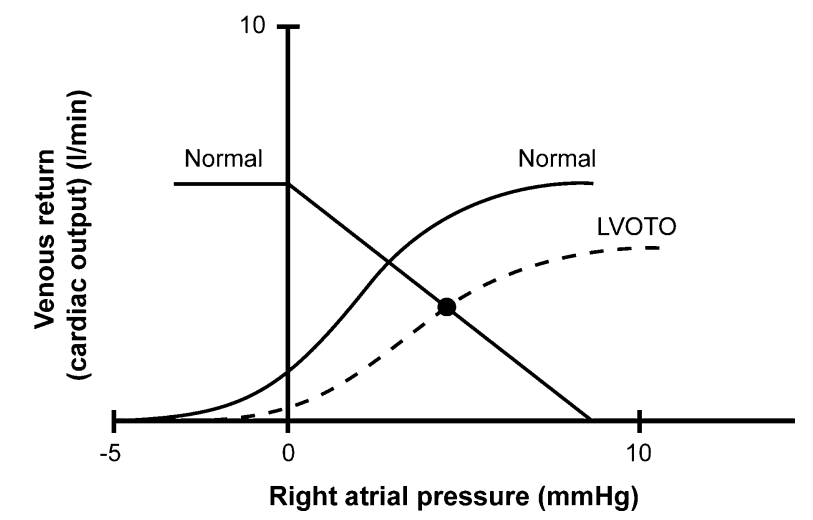

$B_{\text {Pressure-volume relationship }}$ of the left ventricle

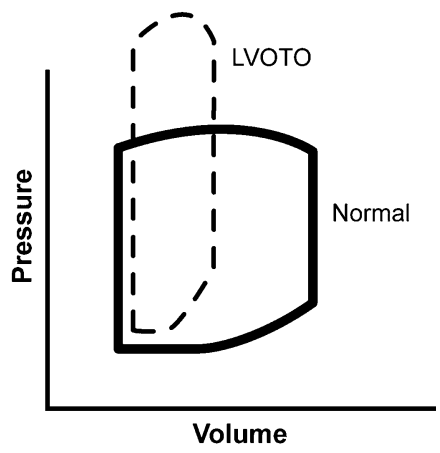

\section{Case 4}

A young male with long-standing diabetes suffered a cardiac arrest secondary to premature coronary artery disease.
Therapeutic hypothermia $\left(34^{\circ} \mathrm{C}\right)$ was induced for $24 \mathrm{hr}$. During rewarming, right ventricular dysfunction was observed and intravenous inotropic therapy was initiated. Shortly thereafter, the patient became hypotensive. His 


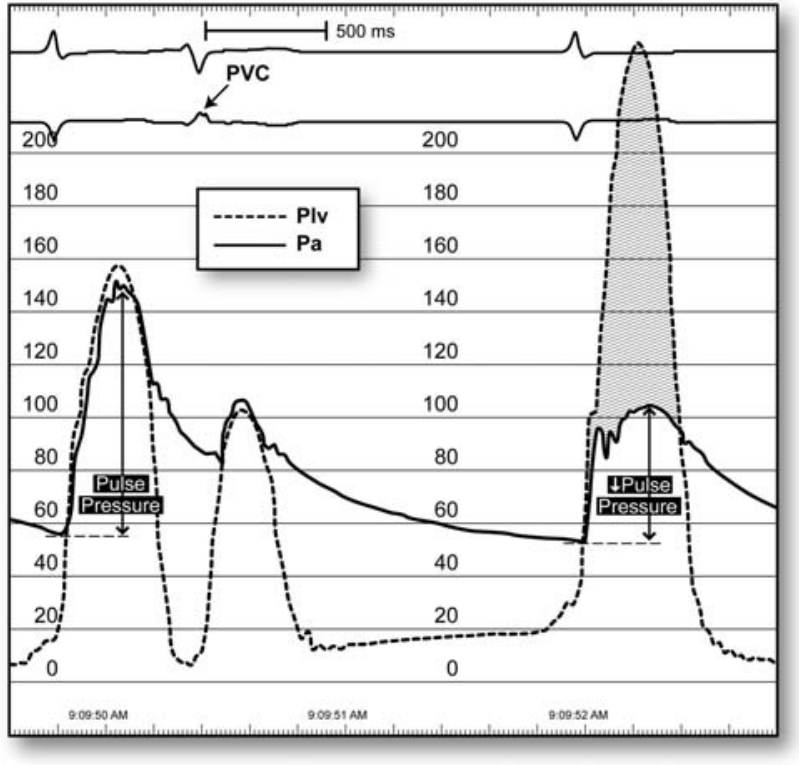

Fig. 7 Brockenbrough-Braunwald reflex in left ventricular outflow tract obstruction (LVOTO). The systolic arterial pressure $(\mathrm{Pa})$ following a premature ventricular complex (PVC) will be reduced. With the compensatory pause that accompanies the PVC, left ventricular pressure (Plv) will have more time to increase and contractility will be enhanced. Normally this will result in a beat with increased systolic pressure. However, in the presence of LVOTO, as the left ventricular contractility is increased, the dynamic obstruction will increase. This explains why the subsequent systolic $\mathrm{Pa}$ will be lower, the diastolic $\mathrm{Pa}$ will be higher, and consequently a reduced pulse pressure will be observed

TEE exam showed LVOTO with SAM and mitral insufficiency (Fig. 4). Inotropic therapy was changed for inhaled prostacyclin and the patient regained normotension and improved right ventricular function.

\section{Discussion}

These cases illustrate four different clinical presentations of the diagnosis of LVOTO. With the ever-increasing use of echocardiography, both in the operating room and in the critical care setting, the diagnosis of LVOTO is being recognized with increasing frequency. Left ventricular outflow tract obstruction can be defined as either fixed or dynamic obstruction to ejection of blood from the left ventricle, with the obstruction located below the aortic valve but sometimes involving regions up to the ventricular apex. The term mid-cavitary or apical obstruction is used to describe the latter. Although the clinical manifestations are similar to those of left ventricular systolic dysfunction, the diagnosis of LVOTO is critical because the treatment and management are based on a very different rationale. Indeed, inotropic support, pharmacological or mechanical afterload reduction, and volume restriction used in heart

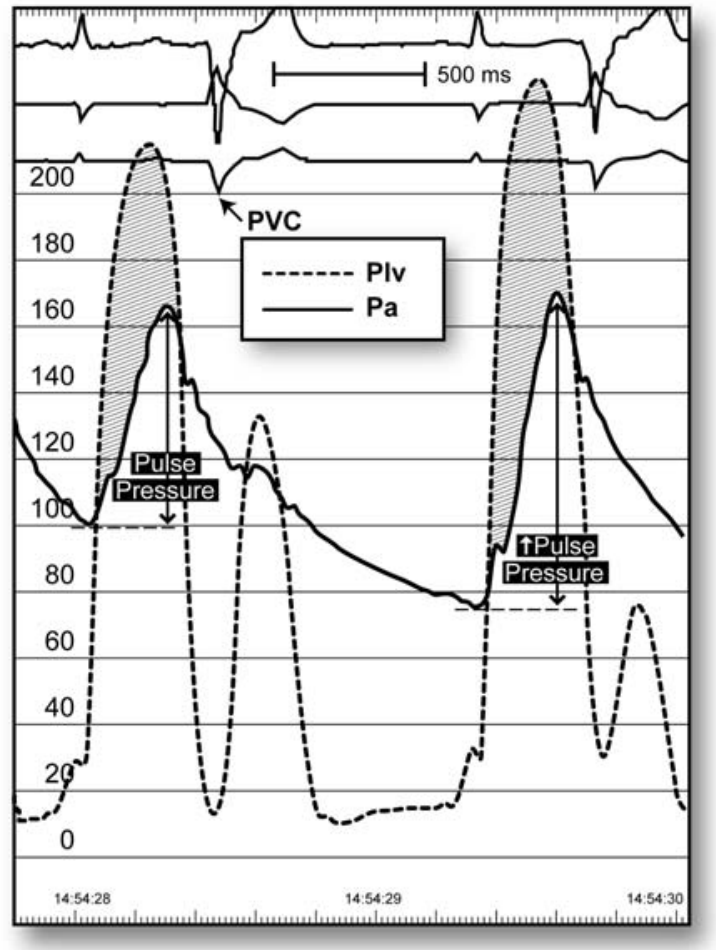

Fig. 8 Aortic stenosis (AS) and a premature ventricular complex (PVC). Note the pulsus tardus of aortic stenosis. The systolic arterial pressure $(\mathrm{Pa})$ following a premature ventricular complex $(\mathrm{PVC})$ is normally increased. With the compensatory pause that accompanies the PVC, left ventricular pressure (Plv) will have more time to increase and contractility will be enhanced. This will result in a beat with increased systolic $\mathrm{Pa}$ and Plv. In the presence of AS, note that the subsequent systolic $\mathrm{Pa}$ is higher, the diastolic $\mathrm{Pa}$ is lower, and consequently an increased pulse pressure will be observed rather than left ventricular outflow tract obstruction where the systolic $\mathrm{Pa}$ is lower, the diastolic $\mathrm{Pa}$ is higher, and consequently a decreased pulse pressure is observed

failure would significantly compromise the hemodynamics of a patient presenting with a low output state from LVOTO. Despite known risk factors for LVOTO (Fig. 5), such as ventricular septal thickness $>13 \mathrm{~mm}$, a long posterior mitral leaflet, an anteriorly displaced coaptation point, and a mitral-aortic angle $>90^{\circ},{ }^{1}$ this condition has been observed in numerous scenarios, and we believe that it can occur in almost every type of hemodynamically unstable patient with markedly reduced left ventricular preload. In the setting of LVOTO, elevated left ventricular filling pressure will be present with flow turbulence in the left ventricular outflow tract. In some patients, this turbulence can lead to suctioning (Venturi effect) or to a drag effect $^{2}$ of the anterior leaflet of the mitral valve into the left ventricular outflow tract during systole, the so-called systolic anterior motion (SAM). This will lead to mitral regurgitation, which is typically eccentric. The consequence will be a reduction in stroke volume and cardiac 
output with an elevated filling pressure (Fig. 6). In the presence of premature ventricular contractions (PVC), a pathognomonic feature of the arterial pressure will be present (as shown in Case 1). This is called the "Brockenbrough-Braunwald" reflex (Fig. 7). ${ }^{3}$ Normally after a PVC, the beat following the compensatory pause will have an increase pulse pressure, even in the presence of aortic stenosis (Fig. 8). In the presence of LVOTO, this normal response will be abolished, and the heartbeat following the compensatory pause will be characterized by a reduced systolic peak and pulse pressure compared with the normal heartbeat (Fig. 7). This occurs because the normal increase in contractility associated with the pause will worsen the LVOTO and result in a reduced systolic pressure. The "Brockenbrough-Braunwald" phenomenon is used by cardiologists in the hemodynamic suite as an end-point for
Fig. 9 Pulsus bisferiens. Typical hemodynamic waveforms in an unstable patient with left ventricular the aspect of the arterial pressure pulse and the components of a pulsus bisferiens. The

echocardiographic equivalence is shown in Fig. 2d outflow tract obstruction. Note

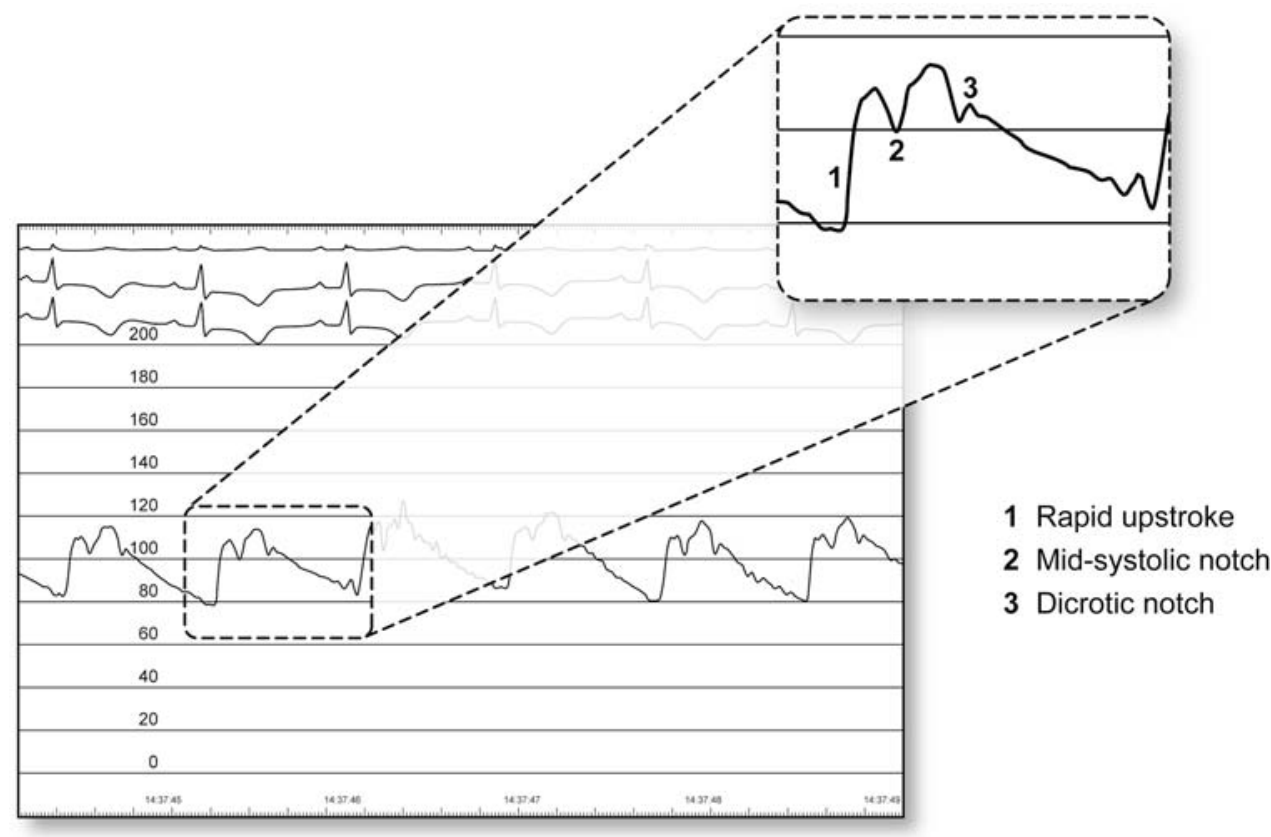

Fig. 10 Proposed approach to hemodynamic instability with left ventricular outflow tract obstruction (LVOTO) (LVOT left ventricular outflow tract; $S A M$ systolic anterior motion; $L V$ left ventricular; $R V$ right ventricular; $R x$ medication)

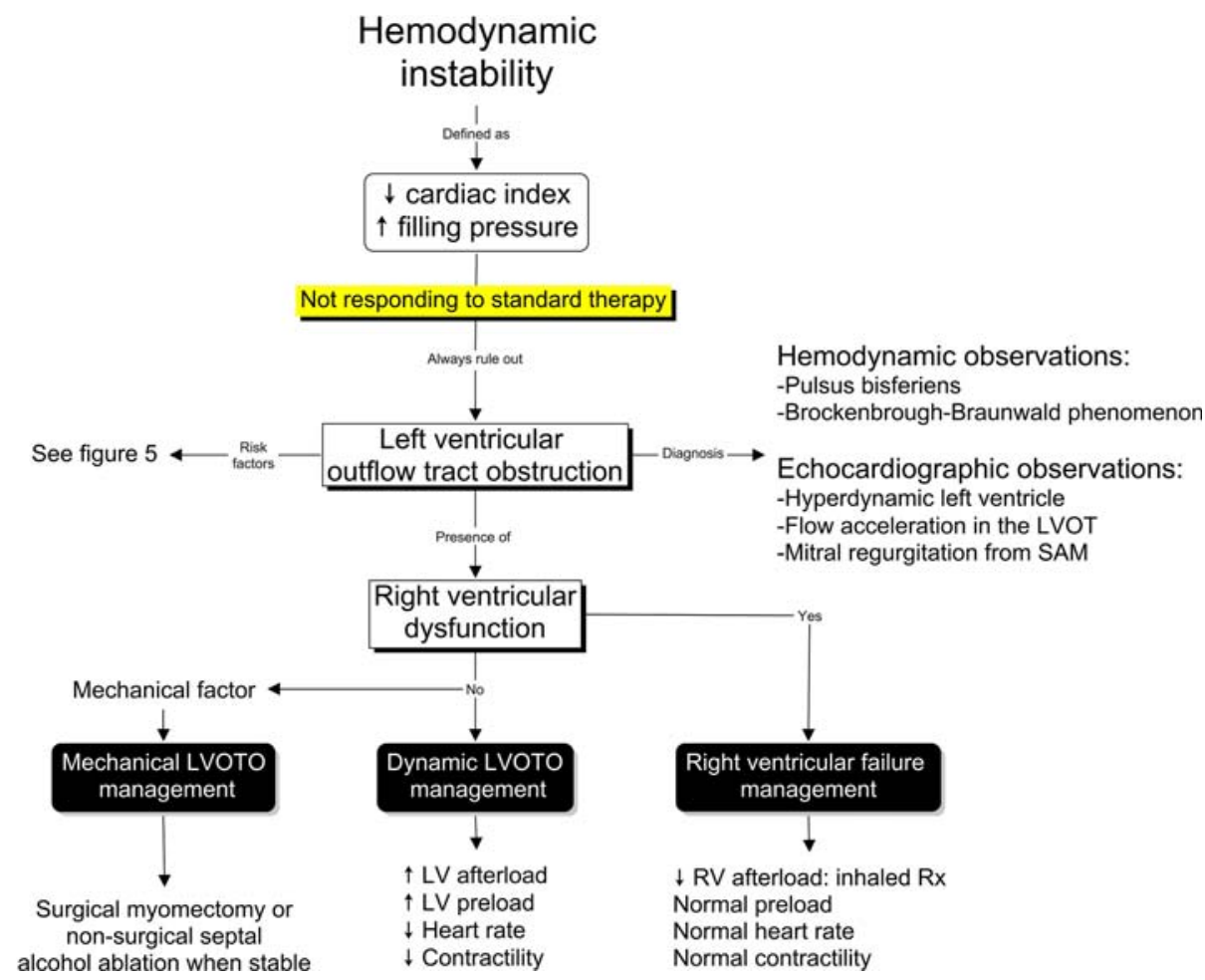


therapeutic alcohol septal ablation in the treatment of hypertrophic cardiomyopathy. Another pathognomonic sign of LVOTO with SAM is the pulsus bisferiens. This is defined as a double systolic pressure peak per cardiac cycle. It is caused initially by the rapid left ventricular ejection and maximum flow through the aortic valve at early systole. As LVOTO appears during mid-systole, the pressure drops. The second peak is secondary to the tidal wave. It is associated with the maximum aortic pressure gradient (Fig. 9). On the TEE exam, the mid-systolic notch can be seen using M-mode (Fig. 2d). It corresponds to the partial closure of the aortic valve at mid-systole. This is followed by the delayed peak pressure associated with the second tidal wave as illustrated in Fig. 4c. Clinically, two types of LVOTO can be present; one is dynamic and the other will have underlying structural anatomical abnormalities such as those observed in hypertrophic obstructive cardiomyopathy (HOCM). In the dynamic form, tachycardia and preload reduction will predispose to LVOTO, as illustrated in Case 3. Using TEE in a series of 61 adults in the intensive care unit with unexplained hypotension for more than one hour, Heidenreich observed that LVOTO was present in $3 \%{ }^{4}$ of patients.

In some patients, right ventricular failure can predispose to LVOTO with SAM, as in Case 4. In this situation, right ventricular dilatation and shifting of the interventricular septum will reduce filling of the left ventricle leading to LVOTO. This is a very difficult situation to manage, as right ventricular dysfunction is associated with poor outcome in numerous scenarios. Inotropic therapy to improve right ventricular function may worsen LVOTO. To improve right ventricular function in such a situation, we have been using inhaled pulmonary vasodilators, such as prostacyclin or nitric oxide, with reduction in LVOTO when present. Finally, extrinsic cardiac obstruction, such as tamponade after cardiac surgery, can also lead to LVOTO. ${ }^{5}$ In these situations, LVOTO will disappear as the underlying cause is removed. An algorithmic approach to LVOTO is presented in Fig. 10.

In conclusion, LVOTO may present in many different clinical situations and should be considered in the differential diagnosis of hemodynamic instability in patients with low cardiac output, high filling pressure, and unresponsiveness to conventional treatment of systolic heart failure. Echocardiography is crucial in its diagnosis and will guide the clinician to the adequate treatment.

Competing interests None declared.

\section{References}

1. Maslow AD, Regan MM, Haering JM, Johnson RG, Levine RA. Echocardiographic predictors of left ventricular outflow tract obstruction and systolic anterior motion of the mitral valve after mitral valve reconstruction for myxomatous valve disease. J Am Coll Cardiol 1999; 34: 2096-104.

2. Sherrid MV, Gunsburg DZ, Moldenhauer S, Pearle G. Systolic anterior motion begins at low left ventricular outflow tract velocity in obstructive hypertrophic cardiomyopathy. J Am Coll Cardiol 2000; 36: 1344-54.

3. Brockenbrough EC, Braunwald E, Morrow AG. A hemodynamic technic for the detection of hypertrophic subaortic stenosis. Circulation 1961; 23: 189-94.

4. Heidenreich PA, Stainback RF, Redberg RF, Schiller NB, Cohen NH, Foster E. Transesophageal echocardiography predicts mortality in critically ill patients with unexplained hypotension. J Am Coll Cardiol 1995; 26: 152-8.

5. Denault AY, Couture P, Buithieu J, Tardif JC. Transesophageal Echocardiography Multimedia Manual: A Perioperative Transdisciplinary Approach. Marcel Dekker; 2005. 